TITLE:

\title{
Dislocation formation in two-phase alloys
}

$\operatorname{AUTHOR}(\mathrm{S}):$

Minami, A; Onuki, A

\section{CITATION:}

Minami, A ... [et al]. Dislocation formation in two-phase alloys. PHYSICAL REVIEW B 2004, 70(18): 184114.

ISSUE DATE:

2004-11

URL:

http://hdl.handle.net/2433/50028

RIGHT:

Copyright 2004 American Physical Society 
PHYSICAL REVIEW B 70, 184114 (2004)

\title{
Dislocation formation in two-phase alloys
}

\author{
Akihiko Minami and Akira Onuki \\ Department of Physics, Kyoto University, Kyoto 606-8502, Japan
}

(Received 25 June 2004; published 18 November 2004)

\begin{abstract}
A phase field model is presented to study dislocation formation (coherency loss) in two-phase binary alloys. In our model the elastic energy density is a periodic function of the shear and tetragonal strains, which allows multiple formation of dislocations. The composition is coupled to the elastic field twofold via lattice misfit and via composition dependence of the elastic moduli. By numerically integrating the dynamic equations in two dimensions, we find that dislocations appear in pairs in the interface region and grow into slips. One end of each slip glides preferentially into the softer region, while the other end remains trapped at the interface. Under uniaxial stretching at deep quenching, slips appear in the softer region and do not penetrate into the harder domains, giving rise to a gradual increase of the stress with increasing applied strain in plastic flow.
\end{abstract}

DOI: 10.1103/PhysRevB.70.184114

PACS number(s): 62.20.Fe, 64.70.Kb, 81.40.Cd

\section{INTRODUCTION}

In crystalline solids many kinds of phase transformations are strongly influenced by the elastic field. ${ }^{1-4}$ Since the work by $\mathrm{Cahn}^{5,6}$ most theoretical studies have been focused on the coherent case in which the lattice planes are continuous through the interfaces. In the incoherent case, on the other hand, dislocations appear around the interfaces and the continuity is lost partially or even completely. Such incoherent microstructures emerge in various alloys when the lattice constants or the crystalline structures of the two phases are not close. ${ }^{7-10}$ Moreover, they are produced in plastic flow because dislocations generated tend to be trapped at the interfaces. In particular, coherency loss has been extensivesly studied in the presence of $\gamma^{\prime}$ precipitates of the $L 1_{2}$ structure. ${ }^{9,10}$

Theory for the incoherent case is much more difficult than for the coherent case, obviously because the effects cannot be adequately described within the usual linear elasticity theory. ${ }^{11}$ The aim of this paper is hence to present a simple mathematical model reasonably describing the incoherent effects in binary alloys. Use will be made of a recent nonlinear elasticity theory of plastic flow by one of the present authors. ${ }^{12}$

A number of authors have studied composition changes around dislocations fixed in space and time, which lead to a compositional Cottrell atmosphere ${ }^{13}$ or preferential nucleation around a dislocation. ${ }^{14,15}$ As recent numerical work in two dimensions, phase separation has been studied by Léonard and Desai ${ }^{16}$ and by $\mathrm{Hu}$ and $\mathrm{Chen}^{17}$ using a continuum Ginzburg-Landau or phase field model in the presence of fixed dislocations. In these papers, dislocations preexist as singular objects before composition changes. We also mention atomistic simulations of dislocation motion influenced by diffusing solutes ${ }^{18}$ or by precipitated domains. ${ }^{19}$

Mechanical properties of two-phase solids are very different from those of one-phase solids. ${ }^{7,13,20}$ In the presence of precipitated domains, dislocations can be pinned at the interface regions and networks of high-density dislocations can be formed preferentially in softer regions after deformations. ${ }^{9}$ These effects are very complex but important in technology. Our simulations will give some insights on the behavior of dislocations in two-phase states.
This paper is organized as follows. In Sec. II we will present the free energy functional for the composition and the elastic field, in which the elastic energy density is a periodic function of the tetragonal and shear strains and the composition is coupled to the elastic field. In Sec. III, we will construct dynamical equations. In Sec. IV, numerical results will be given on the dislocation formation around domains and on the stress-strain relations under uniaxial stretching.

\section{FREE ENERGY FUNCTIONAL}

We consider a binary alloy consisting of two components $A$ and $B$ neglecting vacancies and interstitials. The compositions $c_{A}$ and $c_{B}$ of the two components satisfy $c_{A}+c_{B}=1$. In real metallic alloys undergoing a phase transition, there can be a change in the atomic configuration within unit cells as well as in the overall composition, resulting in ordered domains with the so-called $L 1_{0}$ or $L 1_{2}$ structure. ${ }^{2,4,21}$ However, in this paper, the composition difference is the sole order parameter

$$
\psi=c_{A}-c_{B},
$$

for simplicity. The other variables representing the orderdisorder phase transition are neglected. Then $\psi$ is in the range $-1 \leqq \psi \leqq 1$ and

$$
c_{A}=\frac{1}{2}(1+\psi), \quad c_{B}=\frac{1}{2}(1-\psi) .
$$

In our free energy $F=\int d \boldsymbol{r} f$ the order parameter $\psi$ and the elastic displacement vector $\boldsymbol{u}=\left(u_{x}, u_{y}\right)$ are coupled. The free energy density $f$ is of the form

$$
f=f_{\mathrm{BW}}(\psi)+\frac{C}{2}|\nabla \psi|^{2}+\alpha e_{1} \psi+f_{\mathrm{el}} .
$$

The first term is the Bragg-Williams free energy density expressed as ${ }^{4}$ 


$$
\frac{v_{0}}{k_{\mathrm{B}} T} f_{\mathrm{BW}}=\frac{1+\psi}{2} \ln (1+\psi)+\frac{1-\psi}{2} \ln (1-\psi)-T_{0} \psi^{2} / 2 T,
$$

where $v_{0}$ is the volume of a unit cell representing the atomic volume, $T_{0}$ is the mean-field critical temperature in the absence of the coupling to the elastic field. If $|\psi| \ll 1$, we obtain the Landau expansion $v_{0} f_{\mathrm{BW}} / k_{\mathrm{B}} T=\left(1-T_{0} / T\right) \psi^{2} / 2+\psi^{4} / 24$ $+\cdots$. However, we will not use this expansion form because we are interested in the deeply quenched case. The second term in Eq. (2.3) is the gradient term where $C$ is a positive constant. The parameter $\alpha$ represents the strength of the coupling between the composition and the dilation strain $e_{1}$ $=\nabla \cdot \boldsymbol{u}$. This coupling arises in the presence of a difference in the atomic sizes of the two species and is consistent with the empirical fact that the lattice constant changes linearly as a function of the average composition in many one-phase alloys (Vegard law). It gives rise to a difference in the lattice constants of the two phases in phase separation (lattice misfit). It also explains a composition inhomogeneity (Cottrell atmosphere in one-phase states or precipitate in two-phase states) around a dislocation.

In two dimensions $f_{\mathrm{el}}$ depends on the following strains

$$
\begin{aligned}
& e_{1}=\nabla_{x} u_{x}+\nabla_{y} u_{y}, \\
& e_{2}=\nabla_{x} u_{x}-\nabla_{y} u_{y}, \\
& e_{3}=\nabla_{y} u_{x}+\nabla_{x} u_{y},
\end{aligned}
$$

where $\nabla_{x}=\partial / \partial x$ and $\nabla_{y}=\partial / \partial y$. The elastic displacement $\boldsymbol{u}$ is measured in a reference one-phase state at the critical composition. We call $e_{2}$ the tetragonal strain and $e_{3}$ the shear strain. In this paper we use a nonlinear elastic energy density of the form

$$
f_{\mathrm{el}}=\frac{1}{2} K e_{1}^{2}+\Phi\left(\psi, e_{2}, e_{3}\right) .
$$

The first term represents the elastic energy due to dilation with $K$ being the bulk modulus. The second term arises from anisotropic shear deformations defined for arbitrary values of $e_{2}$ and $e_{3}$. Assuming a square lattice structure, ${ }^{12}$ we set

$$
\Phi=\frac{\mu_{2}}{4 \pi^{2}}\left[1-\cos \left(2 \pi e_{2}\right)\right]+\frac{\mu_{3}}{4 \pi^{2}}\left[1-\cos \left(2 \pi e_{3}\right)\right] .
$$

The principal crystal axes are along or make angles of $\pm \pi / 4$ with respect to the $x$ or $y$ axis. In Fig. 1 we plot $\Phi$ as a function of $e_{2}$ and $e_{3}$ for the case $\mu_{2}=\mu_{3}=\mu_{0}$ in units of $\mu_{0}$. If the system is homogeneous, elastic stability is attained for $\partial^{2} \Phi / \partial e_{2}^{2}>0$ and $\partial^{2} \Phi / \partial e_{3}^{2}>0$ or in the regions $\left|e_{2}-n\right|<1 / 4$ and $\left|e_{3}-m\right|<1 / 4$ with $n$ and $m$ being integer values. ${ }^{12}$

For small strains $\left|e_{2}\right| \ll 1$ and $\left|e_{3}\right| \ll 1$, it follows the usual standard form ${ }^{11}$

$$
\Phi \cong \frac{1}{2} \mu_{2} e_{2}^{2}+\frac{1}{2} \mu_{3} e_{3}^{2},
$$

in the linear elasticity theory. Therefore,

$$
\mu_{2}=\frac{1}{2}\left(C_{11}-C_{12}\right), \mu_{3}=C_{44},
$$

in terms of the usual elastic moduli $C_{11}, C_{12}$, and $C_{44} \cdot{ }^{11} \mathrm{In}$ the original theory ${ }^{5}$ the isotropic linear elasticity with con-

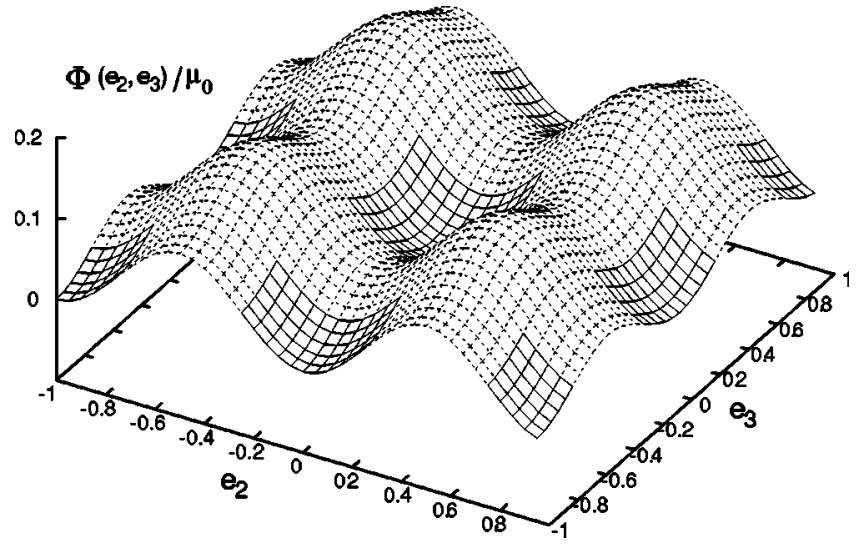

FIG. 1. Normalized shear deformation energy $\Phi\left(e_{2}, e_{3}\right) / \mu_{0}$ for the case $\mu_{2}=\mu_{3}=\mu_{0}$. The elastically stable regions are meshed with solid lines on the surface.

stant $\mu_{2}=\mu_{3}$ was assumed. Subsequent theories treated the case of the cubic linear elasticity with constant $\mu_{2}$ and $\mu_{3}{ }^{2-4,6,22}$ In the present paper, while $K$ is a constant, $\mu_{2}$ and $\mu_{3}$ depend on the composition as

$$
\mu_{2}=\mu_{20}+\mu_{21} \psi, \quad \mu_{3}=\mu_{30}+\mu_{31} \psi .
$$

If $\mu_{21}>0$ and $\mu_{31}>0$, the regions with larger (smaller) $\psi$ are harder (softer) than those with smaller (larger) $\psi$. It is known that this elastic inhomogeneity gives rise to asymmetric elastic deformations in two-phase structures and eventual pinning of the domain growth. ${ }^{4,23,24}$

In our theory $\Phi\left(\psi, e_{2}, e_{3}\right)$ in Eq. (2.7) is the simplest periodic function of $e_{2}$ and $e_{3}$ with period 1 . The periodicity arises from the fact that the square lattice is invariant with respect to a slip of the crystal structure by a unit lattice constant along a line parallel to the $x$ or $y$ axis. Notice that, under rotation of the reference frame by $\theta, e_{2}$ and $e_{3}$ are changed to $e_{2}^{\prime}$ and $e_{3}^{\prime}$, respectively, with ${ }^{12}$

$$
\begin{gathered}
e_{2}^{\prime}=e_{2} \cos 2 \theta+e_{3} \sin 2 \theta, \\
e_{3}^{\prime}=-e_{2} \sin 2 \theta+e_{3} \cos 2 \theta .
\end{gathered}
$$

For $\theta=\pi / 2$ we have $e_{2}^{\prime}=-e_{2}$ and $e_{3}^{\prime}=-e_{3}$, so $f_{\text {el }}$ in Eq. (2.7) remains invariant. For $\theta=\pi / 4$ we have $e_{2}^{\prime}=e_{3}$ and $e_{3}^{\prime}=-e_{2}$ and recognize that the roles of tetragonal and shear strains are exchanged. For $\mu_{2}=\mu_{3}$, the linear elasticity in Eq. (2.8) becomes isotropic, but the nonlinear elasticity is still anisotropic [from the fourth-order terms in the expansion of $\Phi$ in Eq. (2.7) in powers of $e_{2}$ and $e_{3}$ ].

The elastic stress tensor $\stackrel{\leftrightarrow}{\sigma}=\left\{\sigma_{i j}\right\}$ is expressed as

$$
\begin{gathered}
\sigma_{x x}=K e_{1}+\alpha \psi+\mu_{2} \sin \left(2 \pi e_{2}\right) / 2 \pi, \\
\sigma_{y y}=K e_{1}+\alpha \psi-\mu_{2} \sin \left(2 \pi e_{2}\right) / 2 \pi, \\
\sigma_{x y}=\sigma_{y x}=\mu_{3} \sin \left(2 \pi e_{3}\right) / 2 \pi .
\end{gathered}
$$

In the linear elasticity, $\sin \left(2 \pi e_{2}\right) / 2 \pi$ and $\sin \left(2 \pi e_{3}\right) / 2 \pi$ are replaced by $e_{2}$ and $e_{3}$, respectively. Notice the relation 


$$
\nabla \cdot \stackrel{\leftrightarrow}{\sigma}=-\frac{\delta}{\delta u} F,
$$

where $\psi$ is fixed in the functional derivative $\delta F / \delta u$.

The mechanical equilibrium condition $\nabla \cdot \stackrel{\leftrightarrow}{\sigma}=\mathbf{0}$ is equivalent to the extremum condition $\delta F / \delta \boldsymbol{u}=\mathbf{0}$. In the coherent case this condition may be assumed even in dynamics. In fact, using this condition in the linear elasticity, the elastic field has been expressed in terms of $\psi$ in the previous theories (see the appendix). ${ }^{1-6}$ We then find the following. (i) The typical strain around domains is given $b^{4}$

$$
e_{0}=\alpha \Delta c / L_{0},
$$

where $\Delta c=\Delta \psi / 2$ is the composition difference between the two phases and

$$
L_{0}=K+\mu_{20}
$$

is the longitudinal elastic modulus. This strain needs to be small ( $e_{0}<1 / 4$ approximately) as long as the system stays in the coherent regime. (ii) As will be shown in the the Appendix, in the limit of weak cubic elasticity and weak elastic inhomogeneity, one-phase states become linearly unstable for $k_{\mathrm{B}}\left[T-T_{0}+T\langle\psi\rangle^{2} / 2\right] / v_{0}<\alpha^{2} / L_{0}$. At the critical composition $\langle\psi\rangle=0$ this condition becomes $T<T_{\mathrm{s}}$ with

$$
T_{\mathrm{s}}=T_{0}+v_{0} \alpha^{2} / L_{0} k_{\mathrm{B}} .
$$

(iii) Furthermore, Eq. (A.5) suggests that the typical domain size in steady pinned states is a decreasing function of the quench depth $T_{\mathrm{s}}-T$.

\section{DYNAMIC EQUATIONS}

In the incoherent case the mechanical equilibrium does not hold around dislocation cores when dislocations are created and when they are moving. ${ }^{12}$ We thus need to set up the dynamic equation for the elastic displacement $\boldsymbol{u}$. In this paper the lattice velocity $\boldsymbol{v}=\partial \boldsymbol{u} / \partial t$ obeys the momentum equation ${ }^{11}$

$$
\rho \frac{\partial \boldsymbol{v}}{\partial t}=\eta_{0} \nabla^{2} \boldsymbol{v}+\nabla \cdot \stackrel{\leftrightarrow}{\sigma}
$$

The mass density $\rho$ and the shear viscosity $\eta_{0}$ are constants. We neglect the bulk viscosity term $(\propto \nabla \cdot v)$ in Eq. (3.1) for simplicity. ${ }^{12}$ In our model the sound waves relax owing to this viscous damping and the mechanical equilibrium $\nabla \cdot \overleftrightarrow{\sigma}$ $=\mathbf{0}$ is rapidly attained unless $\eta_{0}$ is very small. Note that the nonlinear terms in Eq. (3.1) are only those in $\sigma_{i j}$ in Eq. (2.12).

The composition obeys the diffusive equation

$$
\frac{\partial \psi}{\partial t}=\nabla \cdot \lambda(\psi) \nabla \frac{\delta F}{\delta \psi} .
$$

The kinetic coefficient depends on $\psi$ as ${ }^{25,26}$

$$
\lambda(\psi)=\lambda_{0}\left(1-\psi^{2}\right)=4 \lambda_{0} c_{A} c_{B},
$$

where $\lambda_{0}$ is a constant. Here $\boldsymbol{u}$ is fixed in the chemical potential difference $\delta F / \delta \psi$, so
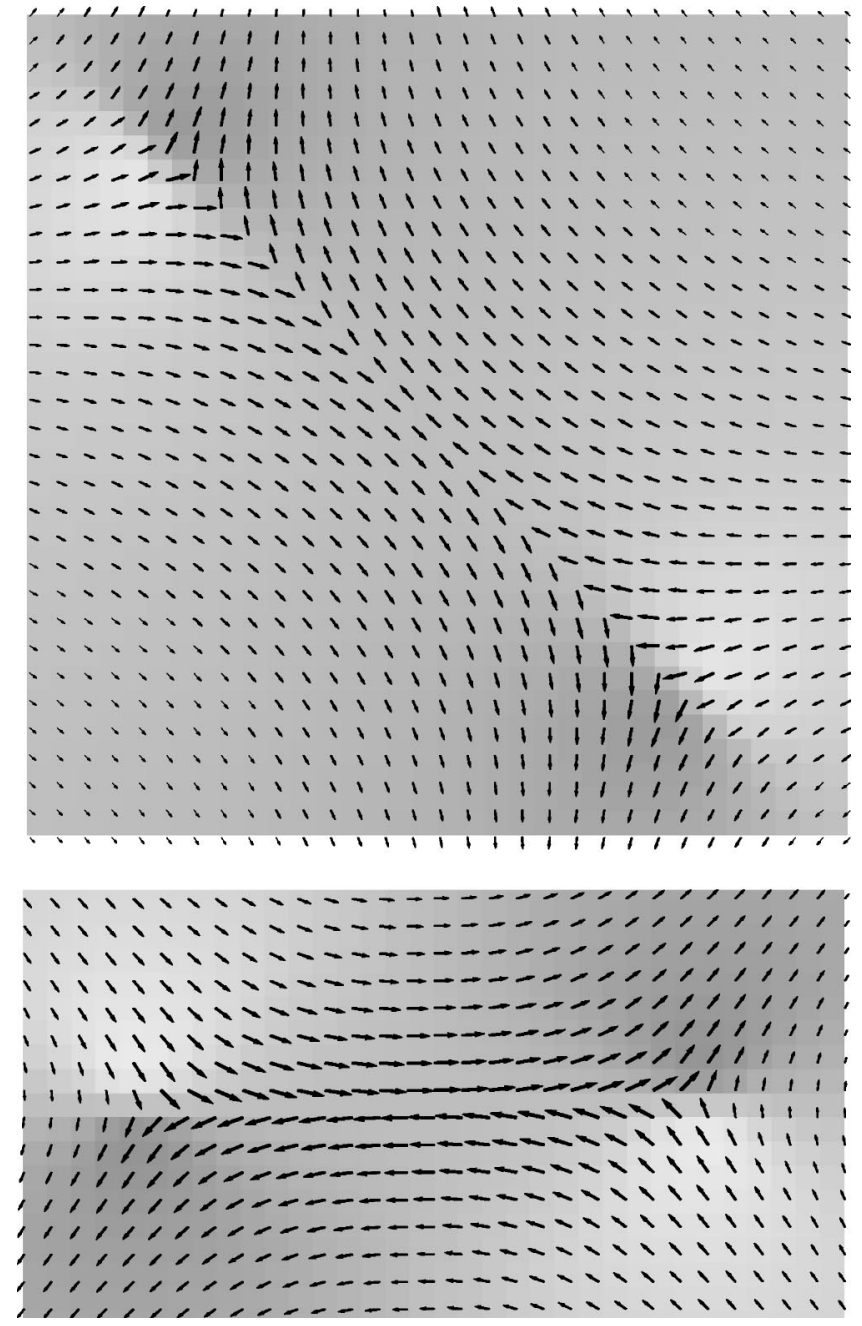

FIG. 2. Displacement vector for a slip (dislocation pair) making an angle of $3 \pi / 4$ (upper plate) and 0 (lower plate) with respect to the $x$ (horizontal) axis in a one-phase steady state at $T / T_{0}=2.5$. The arrows are from the initial position in a perfect crystal to the deformed position. The degree of darkness represents the composition.

$$
\begin{aligned}
\frac{\delta F}{\delta \psi}= & \frac{k_{\mathrm{B}}}{v_{0}}\left[\frac{T}{2} \ln \left(\frac{1+\psi}{1-\psi}\right)-T_{0} \psi\right]-C \nabla^{2} \psi+\alpha e_{1} \\
& +\frac{\mu_{21}}{4 \pi^{2}}\left[1-\cos \left(2 \pi e_{2}\right)\right]+\frac{\mu_{31}}{4 \pi^{2}}\left[1-\cos \left(2 \pi e_{3}\right)\right] .
\end{aligned}
$$

The last two terms arise from the elastic inhomogeneity. If $\lambda(\psi)$ is of the form of Eq. (3.3), the diffusion equation $\partial c_{A} / \partial t=D_{0} \nabla^{2} c_{A}\left(\partial c_{B} / \partial t=D_{0} \nabla^{2} c_{B}\right)$ follows in the dilute limit $c_{A} \rightarrow 0\left(c_{B} \rightarrow 0\right)$ with

$$
D_{0}=\lambda_{0} k_{\mathrm{B}} T v_{0}^{-1},
$$

where the coupling to the elastic field becomes negligible. In usual solid mixtures the diffusion is very slow and vacancies are in many cases crucial for a microscopic description of diffusion. ${ }^{26}$ Effects of such point defects are not treated in the present theory. 


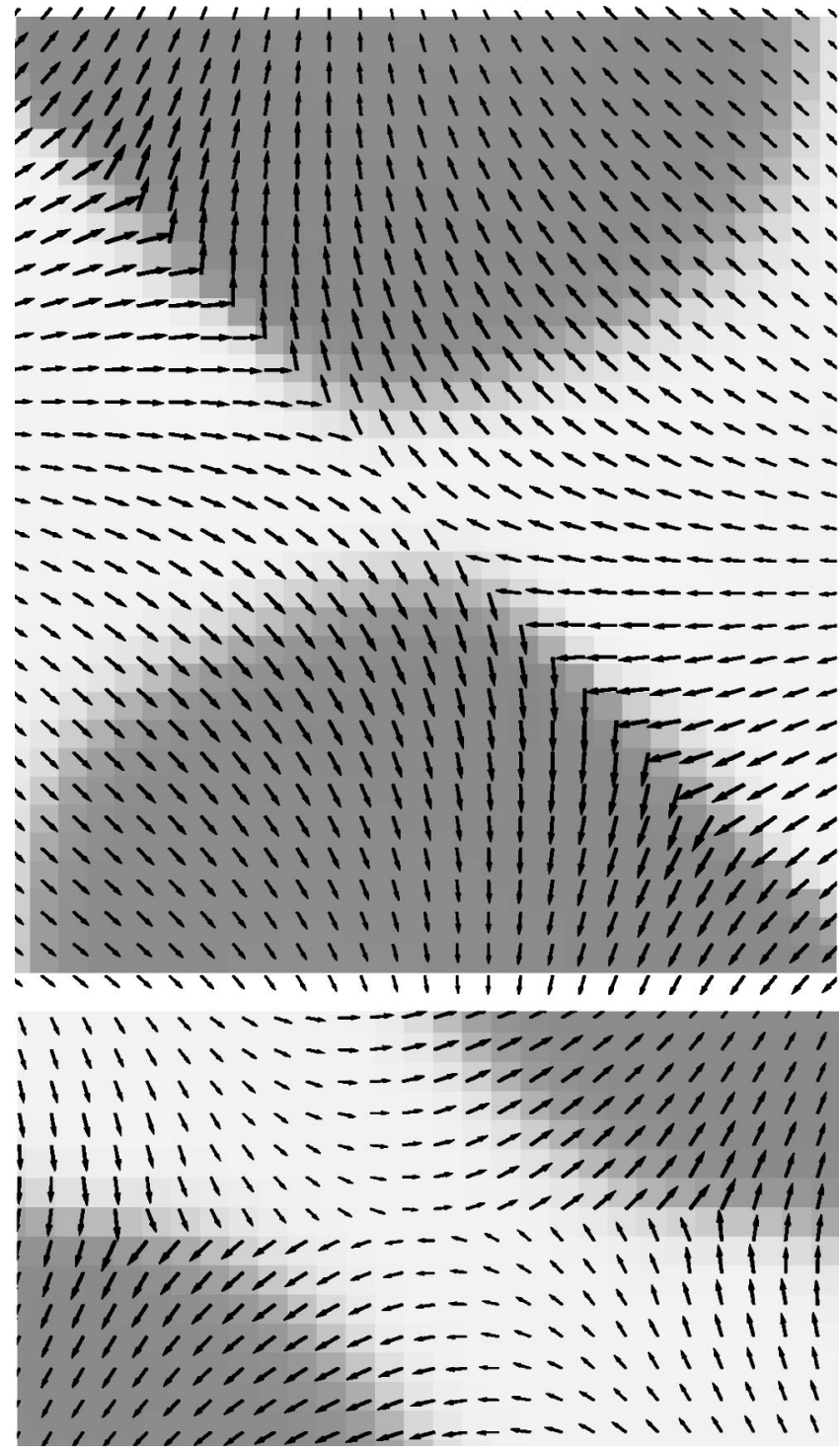

FIG. 3. Displacement vector for a slip (dislocation pair) in a two-phase steady state at $T / T_{0}=1.7$ obtained from the configuration in Fig. 2 after quenching. The arrows are from the initial position in a perfect crystal to the deformed position. The dislocation cores are trapped at the interface regions.

The total free energy $F_{\text {tot }}=F+\int d \boldsymbol{r} \rho \boldsymbol{v}^{2} / 2$ including the kinetic energy then changes in time as

$$
\frac{d}{d t} F_{\text {tot }}=-\int d \boldsymbol{r}\left[\sum_{i j} \eta_{0}\left(\nabla_{i} v_{j}\right)^{2}+\lambda(\psi)\left|\nabla \frac{\delta F}{\delta \psi}\right|^{2}\right] .
$$

Here the surface integrals have been omitted, which vanish if the boundaries are fixed and there is no flux of the atoms from outside (and also if the periodic boundary condition is imposed in simulations). The above time-derivative is nonpositive-definite. As a result, the equilibrium is attained when $\boldsymbol{v}=\nabla \cdot \stackrel{\leftrightarrow}{\sigma}=\mathbf{0}$ and $\delta F / \delta \psi=$ const.

If the lattice is deformed significantly, we should add the convective term $-\nabla(\psi \boldsymbol{v})$ on the right-hand side of Eq. (3.2),

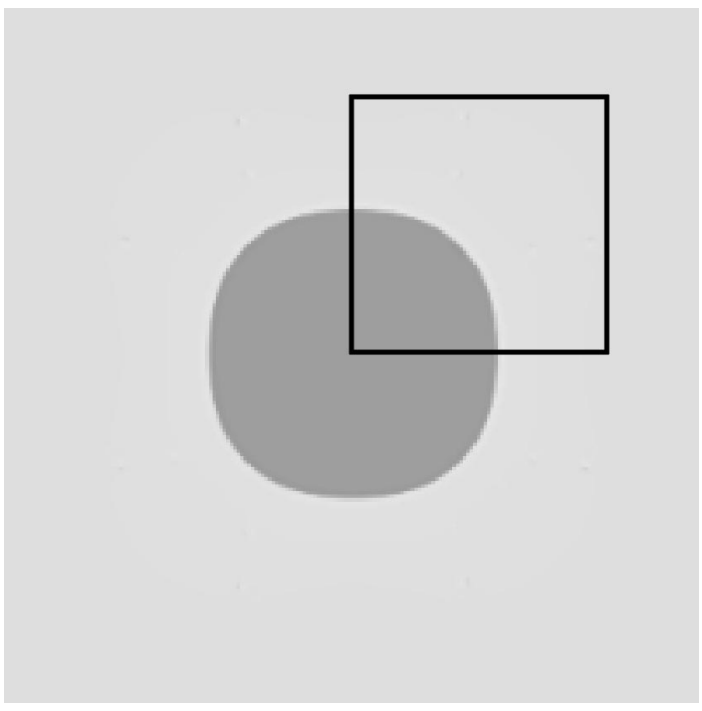

FIG. 4. Hard domain in a steady state at $T / T_{0}=2$. The displacement in the square region will be displayed in Fig. 5 .

treating Eq. (3.2) as the equation in the Euler description. If its presence is assumed, another term of the form $-\psi \nabla \delta F / \delta \psi$ becomes also needed on the right-hand side of Eq. (3.1). With these two terms we again have $d F_{\text {tot }} / d t \leqq 0$. These two terms are well known in critical dynamics of fluids. ${ }^{4}$ However, in our solid case, the magnitude of the displacement $\Delta \boldsymbol{u}=\int_{0}^{t} d t^{\prime} \boldsymbol{v}\left(\boldsymbol{r}, t^{\prime}\right)$ remains small and these two terms give rise to no essential differences in our results at not large applied strains. ${ }^{27}$

Also note that the dynamic Eqs. (3.2) and (3.3) may be treated as Langevin equations with addition of the random noise terms related to the kinetic coefficients $\lambda(\psi)$ and $\eta_{0}$ via the fluctuation-dissipation relations. ${ }^{4}$ In this paper, however, we neglect the random noise, because the thermal energy $k_{\mathrm{B}} T$ will be assumed to be much smaller than the typical energy of elastic deformations.

\section{NUMERICAL RESULTS}

\section{A. Method}

We integrated Eqs. (3.1) and (3.2) in two dimensions on a $256 \times 256$ square lattice. The mesh size $\Delta x$ was set equal to the lattice constant $a$ in the reference state with $\boldsymbol{u}=\mathbf{0}$, so the system length is $L_{0}=256 a$. The vectors $\boldsymbol{u}$ and $\boldsymbol{v}$ are defined at the lattice points $(n, m)$, while the strains, the tensors, and the composition are defined on the middle points $(n+1 / 2, m$ $+1 / 2$ ). These are needed to realize well-defined microscopic slips in our numerical scheme. ${ }^{12}$ The periodic boundary condition was imposed except the simulation of applying uniaxial deformation (Figs. 13 and 14). Because the time scale of $\boldsymbol{u}$ is shorter than that of $\psi$, we integrated Eq. (3.1) using an implicit Crank-Nicolson method. Space and time will be measured in units of $a$ and

$$
\tau_{0}=\left(\rho / \mu_{20}\right)^{1 / 2} a,
$$

respectively, where $\mu_{20}$ is defined by Eq. (2.10) and $\left(\mu_{20} / \rho\right)^{1 / 2}$ is the transverse sound velocity propagating in 


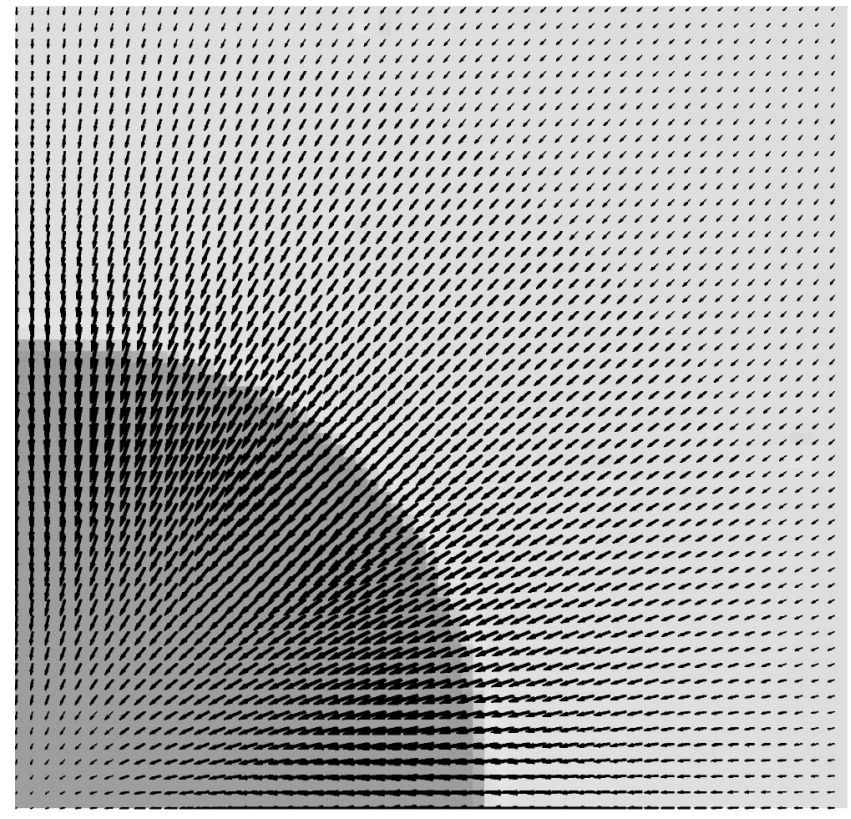

\section{$\boldsymbol{U}_{\text {coh }}$}

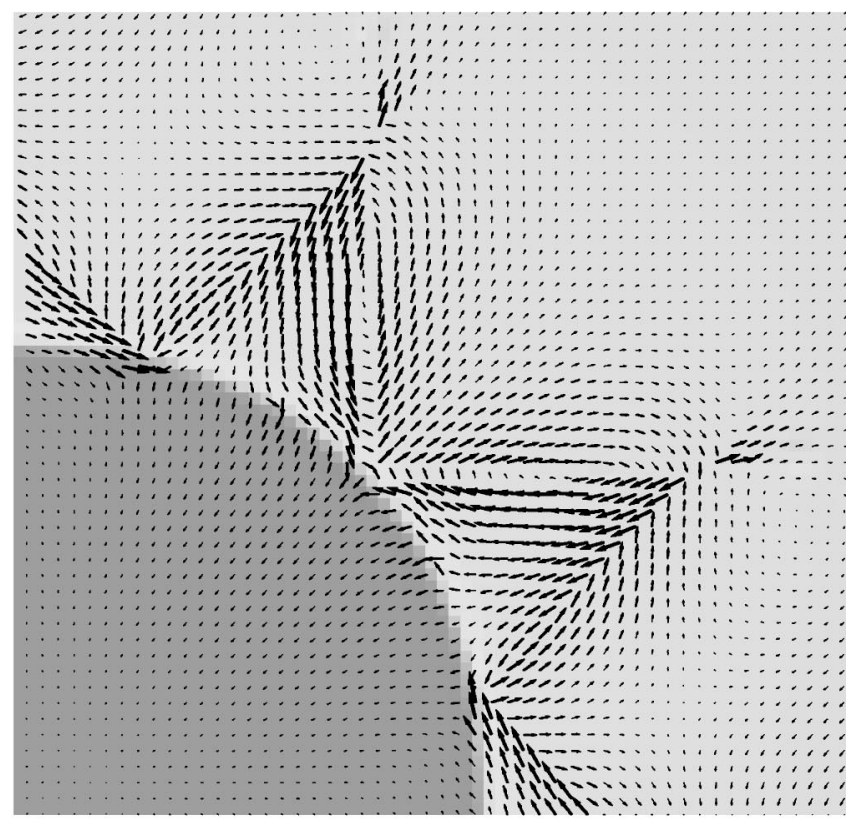

\section{$\boldsymbol{U}-\boldsymbol{U}_{\mathrm{coh}}$}

FIG. 5. Upper plate: Coherent elastic displacement $u_{\text {coh }}$ just before birth of dislocations at deep quenching at $T / T_{0}=1$. Lower plate: Incoherent elastic displacement after appearance of dislocations.

the ${ }^{11}$ direction. The free energies and the free energy densities are measured in units of $\mu_{20} a^{2}$ and $\mu_{20}$, respectively. For simplicity, the scaled time $\tau_{0}^{-1} t$, position vector $a^{-1} \boldsymbol{r}$, and displacement vector $a^{-1} \boldsymbol{u}$ will be written as $t, \boldsymbol{r}$, and $\boldsymbol{u}$, respectively, in the same notation.

In this paper we set $K / \mu_{20}=4.5, \alpha / \mu_{20}=0.6, C / a^{2} \mu_{20}$ $=0.05$ and $k_{\mathrm{B}} T_{0} / v_{0} \mu_{20}=0.05$, where $T_{0}$ is the mean-field critical temperature in Eq. (2.4). Since $T \sim T_{0}$ hereafter, the

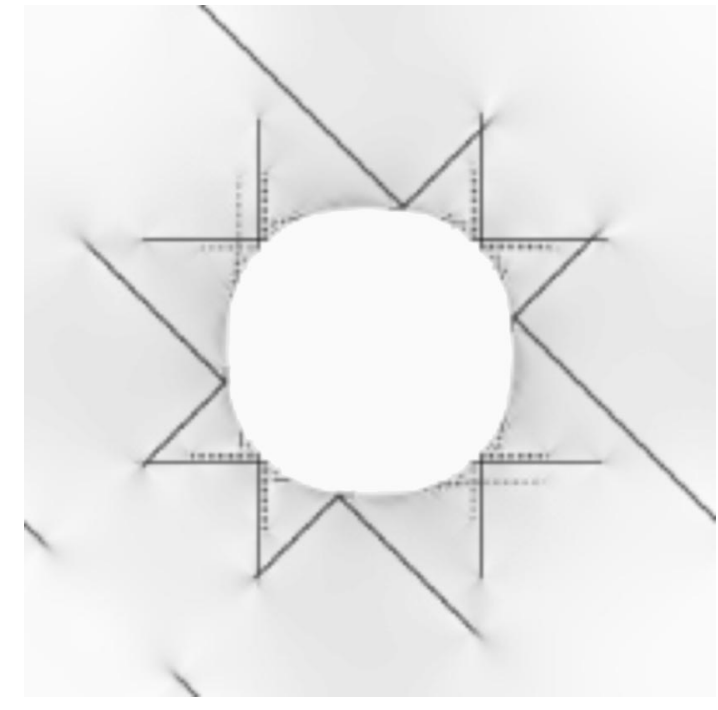

FIG. 6. Snapshot of $e$ in Eq. (4.4) after dislocation formation, which is zero within the hard domain and nonvanishing outside. The slip lines end at dislocations.

elastic energy to create a single slip $\left(\sim \mu_{20} v_{0}\right)$ is much larger than $k_{\mathrm{B}} T$ in our simulations. Furthermore, we assume weak cubic elastic anisotropy with $\mu_{30} / \mu_{20}=1.1$ and moderate elastic inhomogeneity with $\mu_{21}=\mu_{31}=0.6 \mu_{20}$.

The dimensionless kinetic coefficients are given by

$$
\lambda_{0}^{*}=\lambda_{0} \tau_{0} \mu_{20} a^{-2}, \quad \eta_{0}^{*}=\eta_{0} / \tau_{0} \mu_{20} .
$$

We set $\lambda_{0}^{*}=10^{-4}$ and $\eta_{0}^{*}=0.1$. Then,

$$
\lambda_{0}^{*} / \mu_{0}^{*}=D_{0} \rho / \eta_{0} \sim 10^{-3} .
$$

Since the relaxation rate of a sound with wave-number $k$ is $\eta_{0} k^{2} / \rho$, the time scale of $\psi$ becomes longer than that of the elastic field by three orders of magnitude. In real solid alloys, these two time scales are much more distinctly separated, probably except for hydrogen-metal systems where the protons diffuse quickly. ${ }^{4}$

In homogeneous one-phase states we have $e_{2}=e_{3}=0$ and $e_{1}=-\alpha \psi / K$. Here, well known is a parameter $\eta=|\partial a / \partial \psi| / a$ representing the strength of the composition dependence of the lattice constant $a$ in a mixture. ${ }^{5}$ In our case we have $\eta$ $=\alpha / 2 K=0.067$ and the spinodal temperature $T_{\mathrm{s}}$ in Eq. (2.16) becomes 2.31 .

\section{B. Slips and composition changes}

Edge dislocations appear in the form of slips or dipole pairs, ${ }^{12}$ because a single isolated dislocation requires a very large elastic energy. Slips are thus fundamental units of plastic deformations. In Fig. 2 we show the displacement and the composition around typical slips in a one-phase steady state with length $10 \sqrt{2} a$ in the upper plate and $10 a$ in the lower plate. Here we initially prepared a slip given by the linear elasticity theory ${ }^{12}$ at the critical composition $(\langle\psi\rangle=0)$ and let $\boldsymbol{u}$ and $\psi$ relax until the steady state was achieved. The temperature was kept at $T / T_{0}=2.5$ and no phase separation occurred. As in the previous simulations, ${ }^{17,18}$ we can see Cot- 
trell atmospheres around the dislocation cores. The maximum and minimum of $\psi$ at the lattice points close to the dislocation cores are of order \pm 0.6 . Cottrell's result is obtained as follows: Let $T$ be much higher than $T_{0}$ and $\alpha^{2} / L_{0}$ and the gradient term be neglected; then, the condition $\delta F / \delta \psi=$ const. yields $\quad c_{A} /\left(1-c_{A}\right)=$ const. $\exp \left(-U / k_{\mathrm{B}} T\right)$, where $U=v_{0} \alpha e_{1} \cdot{ }^{28}$ In our case the maximum of $|U| / k_{\mathrm{B}} T$ at the lattice points is of order 1 and the accumulation is not very strong.

As a next step, starting with the configuration in Fig. 2, we lowered the temperature to $T / T_{0}=2$ to induce spinodal decomposition. Subsequently the Cottrell atmospheres grew into domains and the dislocation cores stayed at the interface regions. The domain size attained finally was of order $50 a$. Figure 3 illustrates the displacement and the composition in the final steady state, where the maximum and minimum of $\psi$ are about \pm 0.9 . Léonard and Desai ${ }^{16}$ obtained similar composition profiles in spinodal decomposition, where the elastic field of dislocations (given by the linear elasticity theory) was fixed in space and time.

Mathematically, slips in steady states satisfy $\delta F / \delta \boldsymbol{u}=\mathbf{0}$ and $\delta F / \delta \psi=$ const. Without externally applied strains, they are metastable owing to the Peierls potential energy arising from the discreteness of the lattice structure. ${ }^{12}$ Although not discussed in this paper, slips become unstable against expansion or shrinkage with increasing applied strain.

\section{Dislocation formation around a hard domain}

Figure 4 shows a single large hard (A-rich) domain at the center in the coherent condition at shallow quenching $T / T_{0}$ $=2$ after a long equilibration time. Here $\psi$ is about 0.7 inside the domain and about -0.7 outside. Its shape slightly deviates from sphericity owing to the weak cubic anisotropy assumed in this paper. We next performed a second deeper quenching to $T / T_{0}=1$. Subsequent diffusional adjustment of the composition proceeded very slowly, but a discontinuity of the order parameter $\Delta \psi$ about 1.8 was established relatively rapidly across the interface. ${ }^{4}$ As a result, at a time about 1000 after the second quenching, the maximum of $\left|e_{2}\right|$ reached $1 / 4$, the value at the stability limit, in the interface region [see the sentences below Eq. (2.7)]. We then observed formation of dislocations and generation of sound waves emitted from the dislocations. The upper panel of Fig. 5 shows the coherent elastic displacement $\boldsymbol{u}_{\text {coh }}$ just before the dislocation formation, while the lower panel shows the subsequent additional incoherent change $\delta \boldsymbol{u}=\boldsymbol{u}-\boldsymbol{u}_{\text {coh }}$ after a time interval of 1000. The free energy $F$ in the state in the lower panel is smaller than that in the upper panel by 152.9 in units of $\mu_{20} a^{2}$. More details are as folllows: (i) two pairs of dislocation dipoles (four dislocations) appeared simultaneously in a narrow region; (ii) two of them glided preferentially into the softer region forming two slips perpendicular to each other; and (iii) slips collided in many cases and stopped far from the droplet, resulting in a nearly steady elastic deformation. Thus a half of the dislocation cores stayed at the interface and the others were distributed around the domain. These three processes took only a short time of order 100 .

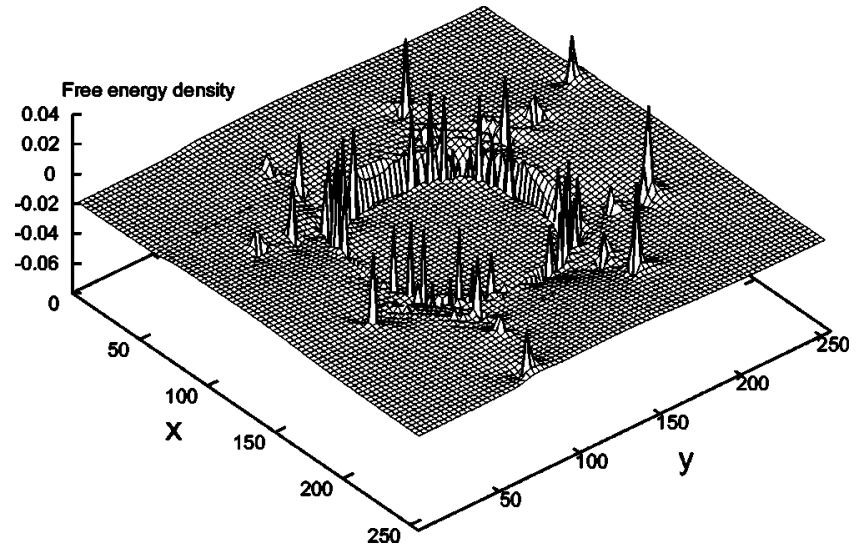

FIG. 7. Snapshot of the free energy density $f$ in Eq. (2.3). The peaks are located near the dislocation cores and the clifflike structure represents the interface free energy density.

After the above dislocation formation at a relatively early stage, the composition changed very slowly. We show three figures at $t=23000$. In Fig. 6 we displays the following strain:

$$
e=\left(e_{2}^{2}+e_{3}^{2}\right)^{1 / 2},
$$

which is invariant with respect to the rotational transformation (2.11). The slips make an angle of $\pm \pi / 4$ with respect to the $x$ axis in the regions with large $\left|e_{2}\right|$ (in the uniaxially deformed regions), while they are parallel to the $x$ or $y$ axis in the corner regions with large $\left|e_{3}\right|{ }^{12} \mathrm{We}$ also notice that the dislocation formation took place with the symmetry axis in the ${ }^{11}$ direction for our special geometry. Figure 7 gives the free energy density $f$ in Eq. (2.3), where the peaks outside the domain represent the dislocation cores. In the interface region it exhibits a clifflike structure arising from the gradient term and higher peaks arising from the dislocation cores. Figure 8 shows the order parameter $\psi$, where we can see Cottrell atmospheres around the dislocation cores surrounding the domain. The system is still transient and there is still

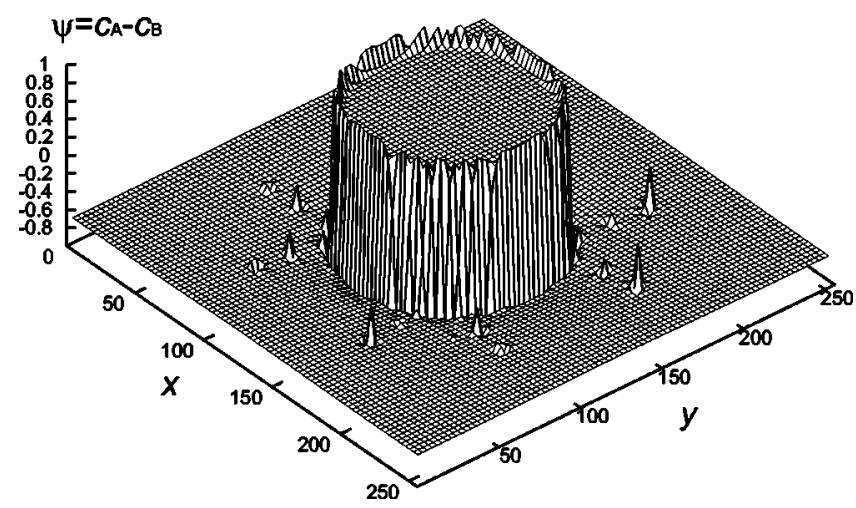

FIG. 8. Snapshot of the order parameter around a hard domain in the incoherent case obtained after a two-step quench. The peak structure at the interface arises because the system away from the interface is still in a transient state. The peaks around the dislocation cores in the outer soft region represent Cottrell atmospheres (but the minima paired are not seen in the figure). 


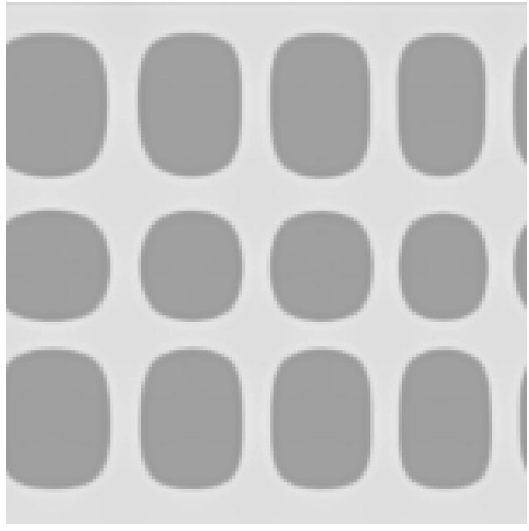

FIG. 9. Domain structure obtained at a shall quench $T / T_{0}=2$ in the coherent condition.

a small composition flux through the interface.

\section{Dislocation formation in a soft network}

Next we examine dislocation formation when hard rectangular domains are densely distributed and wrapped by a percolated soft network. As in Fig. 9, we prepared such a steady domain structure at $T / T_{0}=2$ in the coherent condition. As in the previous simulations, ${ }^{23,24}$ the hard domains (in gray) are elastically isotropic, while the soft network (in white) is mostly uniaxially stretched. That is, in the soft stripes between the two adjacent hard domains, we obtain $e_{2} \sim 0.2$ in the horizontal stripes and $e_{2} \sim-0.2$ in the vertical stripes. We then quenched $T$ to $T / T_{0}=1$ to induce the composition readjustment. Figure 10 displays the resultant time evolution of the total free energy $F=\int d \boldsymbol{r} f$ and the snapshots of $e$ in Eq. (4.4) at the points $\mathrm{A}, \ldots$, and E. It demonstrates that $F$ mainly decreases due to the composition change but sometimes due to appearance and gliding of slips in the soft stripes. Note that the overall composition adjustment occurs slowly on the time scale of $R^{2} / D_{0}=10^{5}-10^{6}$ where $R$ is the domain size. In Fig. 11 we show the displacement $\boldsymbol{u}$ within the square window in $\mathrm{B}, \mathrm{C}$, and $\mathrm{D}$, respectively, while in Fig. 12 the bird views of the free energy density $f$ the square window are given at $t=0$ and 4475 after the second quench. Figure 12 clearly illustrates appearance of the peaks representing the dislocation cores.

\section{E. Uniaxial stretching in two-phase states}

Finally we apply a constant uniaxial deformation to initially coherent states with $\langle\psi\rangle=0$ to induce plastic flow. That is, we set $u_{x}=u_{y}=0$ at the bottom $(y=0)$ and $u_{x}=-u_{y}$ $=\epsilon L_{0} / 2$ at the top $\left(y=L_{0}\right)$. The applied strain rate was fixed at $\dot{\epsilon}=10^{-4}$, so $\epsilon=\dot{\epsilon} t$ with $t$ being the time after application of the deformation. In Fig. 13 we plot the average normal stress $N_{1}$ versus the applied strain $\epsilon$ for $T / T_{0}=3,2.4$, and 2 (upper plate), where

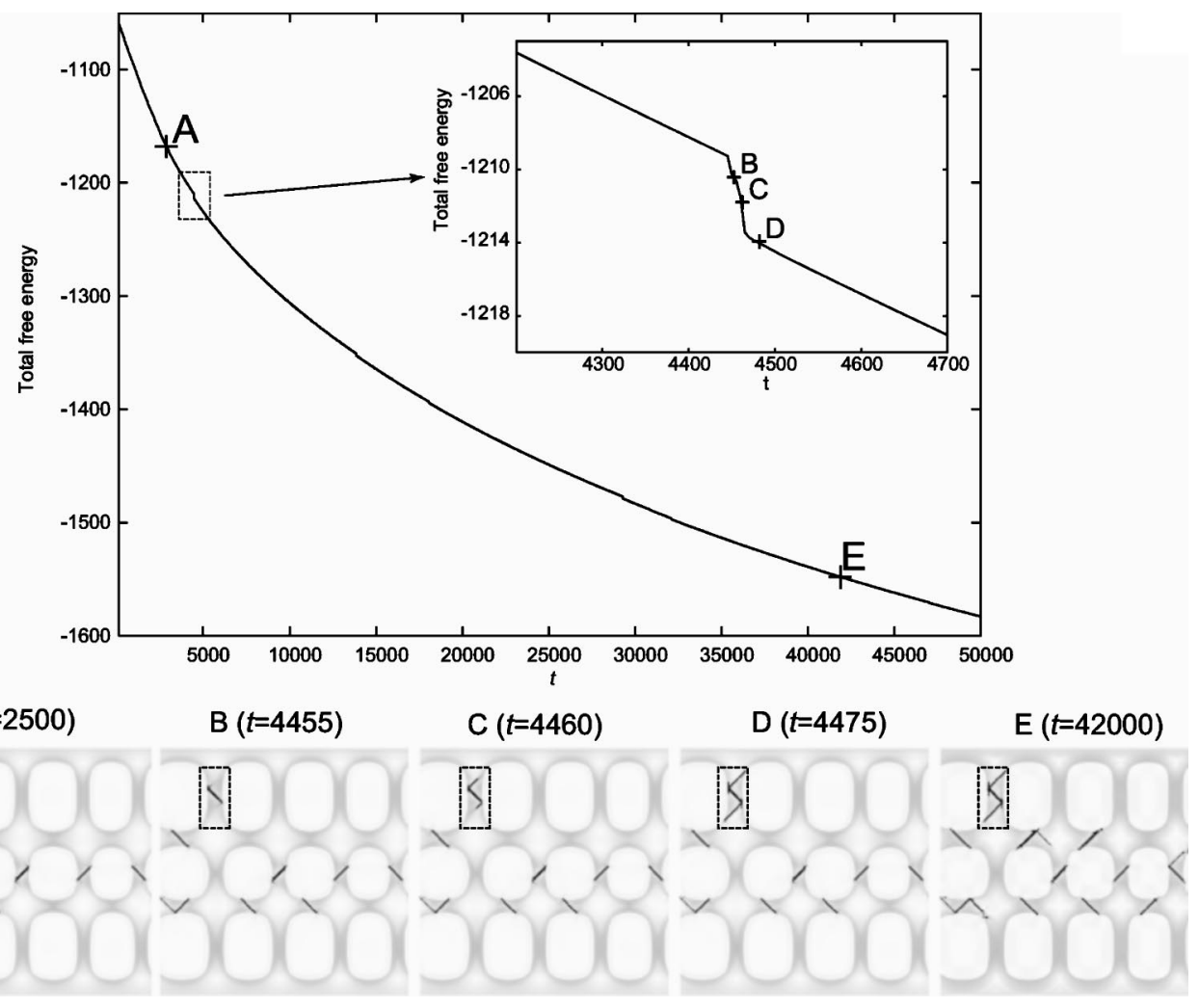

FIG. 10. Relaxation of the total free energy $F$ in units of $\mu_{20} v_{0}=20 k_{\mathrm{B}} T_{0}$ after a two-step quench from $T / T_{0}=2$ to 1 with the initial configuration in Fig. 9. It mostly relaxes due to the gradual composition adjustment, but it sometimes relaxed due to dislocation formation as enlarged in the inset. Snapshots of $e$ at the points $\mathrm{A} \sim \mathrm{E}$ are given in the lower plates. 


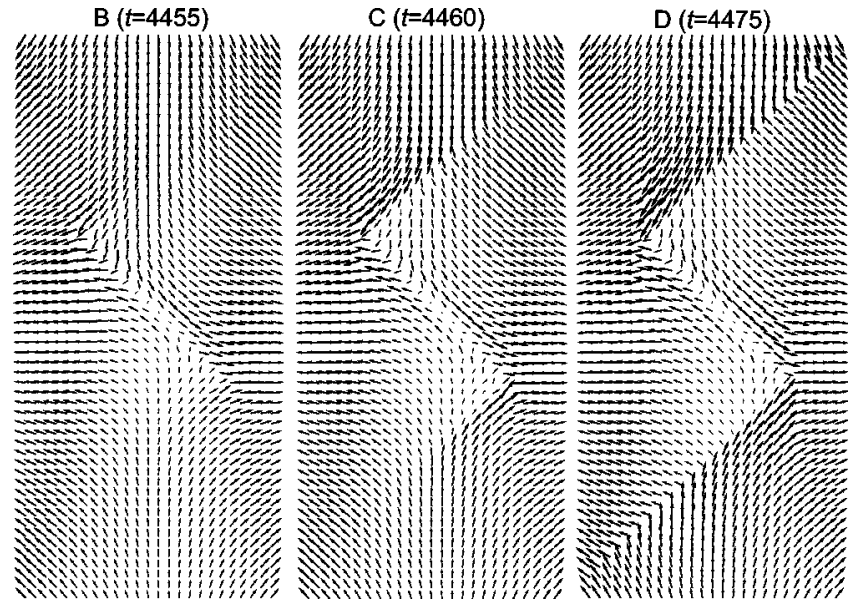

FIG. 11. Elastic displacement $u$ in the marked regions B, C, and D.

$$
N_{1}=\left\langle\sigma_{x x}-\sigma_{y y}\right\rangle=\frac{1}{\pi}\left\langle\mu_{2} \sin \left(2 \pi e_{2}\right)\right\rangle,
$$

where $\langle\cdots\rangle$ denotes taking the spatial average. The snapshots of $e$ in Eq. (4.4) are also given at the points a, b, and c (lower plates). For $T / T_{0}=3$ the system is in a homogeneous onephase state and random numbers with variance 0.01 were assigned to $\psi$ at the lattice points at $t=0$. In the initial state at $T / T_{0}=2.4$ the maximum and minimum of $\psi$ and $e_{2}$ are \pm 0.32 and \pm 0.05 , respectively. At $T / T_{0}=2$ these numbers are mag-
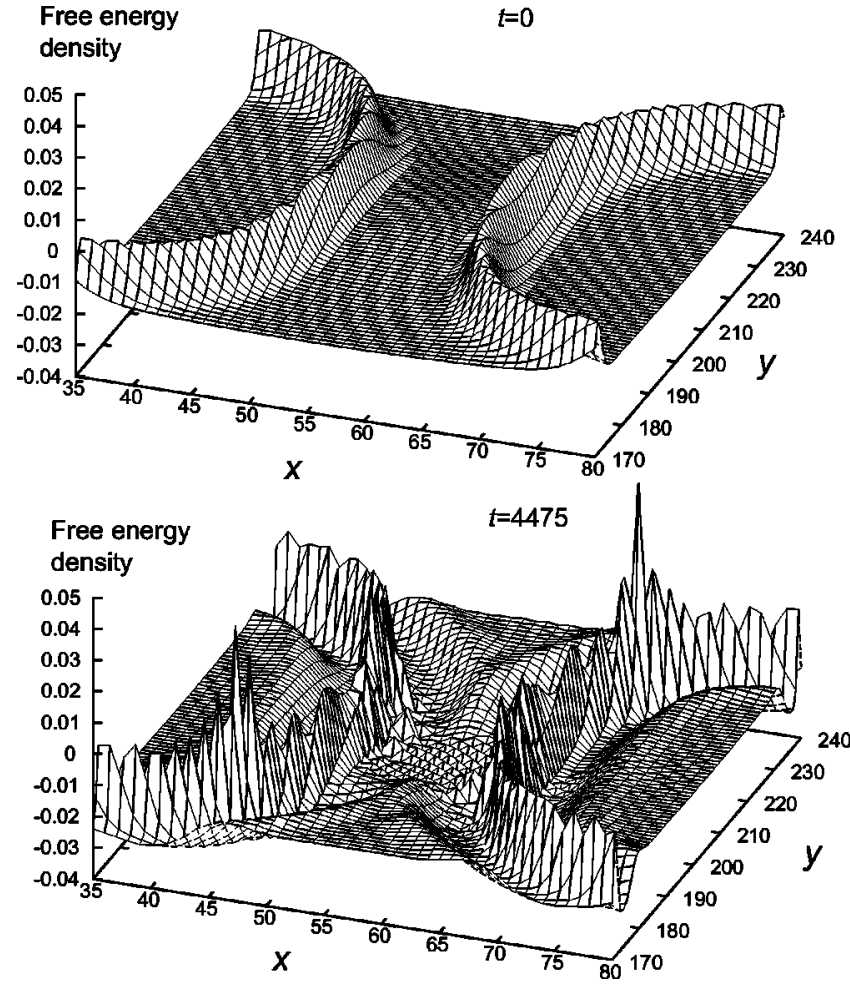

FIG. 12. Bird views of the free energy density $f$ in units of $\mu_{20}=20 k_{\mathrm{B}} T_{0} v_{0}^{-1}$ at $t=0$ and 4475 after the two-step quench in Fig. 10.

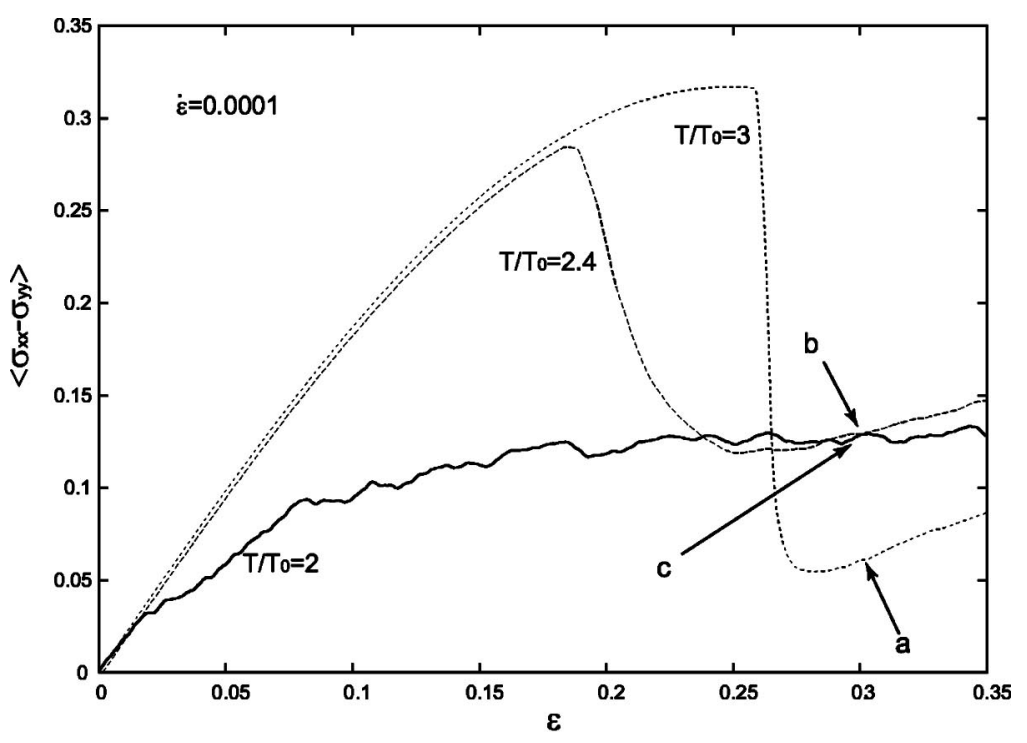

FIG. 13. Stress-strain curves after application of uniaxial stretching $\epsilon=\dot{\epsilon} t$ with $\dot{\epsilon}=10^{-4}$ for $T / T_{0}=3,2.4$, and 2. There is no dislocation at $t$ $=0$. Snapshots of $e$ in Eq. (4.4) at points a, b, and $\mathrm{c}$ are given below, which represent slip patterns in plastic flow.

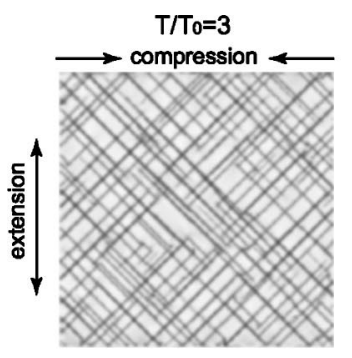

a
$\mathrm{T} / \mathrm{T}_{0}=2.4$

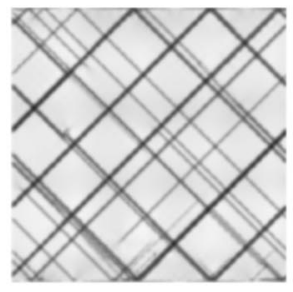

b
$T / T_{0}=2$

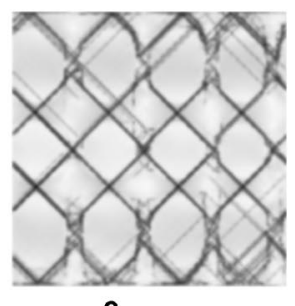

c 


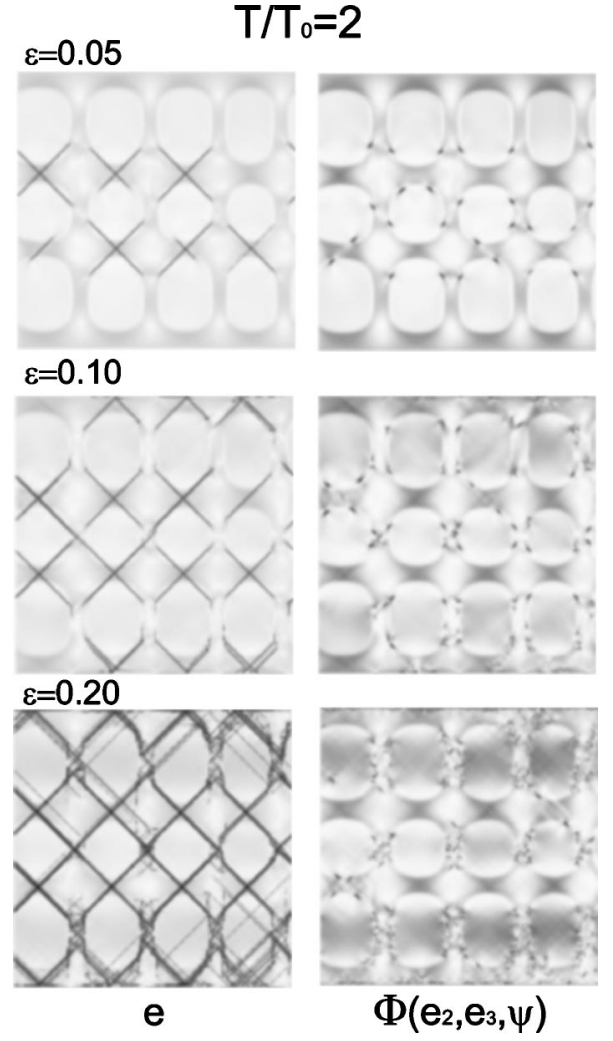

FIG. 14. Snapshots of $e$ in Eq. (4.4) and the shear deformation energy density $\Phi$ in Eq. (2.7) at $T / T_{0}=2$ for $\epsilon=0.05,0.1$, and 0.2 .

nified to \pm 0.75 and \pm 0.20 . All the initial states are coherent without dislocations. For $T / T_{0}=3$ the elastic instability occurs at $\epsilon=1 / 4$ resulting in a fine mesh of slips as in the lower left plate. For $T / T_{0}=2.4$ the onset point of the slip formation is decreased to $\epsilon=0.17$. For $T / T_{0}=3$ the onset is very early at 0.015 , the stress-strain relation exhibits zig-zag behavior upon appearance of slips, and the stress continue to increase on the average (up to the upper bound of $\epsilon$ given by 0.35 in the simulation). Figure 14 consists of snapshots of $e$ in Eq. (4.4) and the shear deformation energy density $\Phi$ in Eq. (2.7) in units of $\mu_{20}$. We can see the quadratic appearance of dislocations at the center of the uniaxially stretched stripes at $\epsilon=0.05$ (top plate), gliding of the dislocations and pinning at the interfaces at $\epsilon=0.1$ (top and middle plates), and thickening of the slips into shear bands at $\epsilon=0.2$ (bottom plate). In our simulations, when soft regions between hard domains are narrow, they can be strongly deformed uniaxially and dislocations often appear at their middle points.

We mention a creep experiment in the presence of high volume fractions of $\gamma^{\prime}$ precipitates, ${ }^{9}$ where softer disordered $\gamma$ regions were observed to be filled with dislocation networks after large deformations.

\section{SUMMARY AND CONCLUDING REMARKS}

In summary, we have presented a coarse-grained phase field model of plastic deformations in two-phase alloys. Though our simulations have been performed in two dimensions, a number of insights into the very complex processes of plasticity have been gained. We mention them and give some remarks.

(1) Performing a two-step quench, we have numerically examined dislocation formation around the interface regions, which occur spontaneously in deeply quenched phase separation. Experimentally, ${ }^{10}$ dislocation formation has been observed around growing $\gamma^{\prime}\left(\mathrm{Al}_{3} \mathrm{Sc}\right)$ precipitates at low volume fractions when the radii exceeded a threshold about $20 \mathrm{~nm}$. Such spontaneous dislocation formation with domain growth has not yet been studied theoretically.

(2) We have found that dislocations glide preferentially into the softer regions with smaller shear moduli and tend to be trapped in the interface regions in agreement with a number of observations. ${ }^{7}$ Theoretically, the composition dependence of the elastic moduli (elastic inhomogeneity) is a crucial ingredient to explain the experiments.

(3) We have applied uniaxial strain to create multiple slips in two-phase alloys which were initially in the coherent condition. The dislocation formation starts in the mostly stretched middle points of the soft stripes. A stress-strain curve in Fig. 13 at deep quenching is very different from the curves in one-phase states. In real two-phase alloys, a similar monotonic increase of the stress without overshoot has been observed, but a considerable amount of defects should preexist in such experiments particularly in work-hardened samples. ${ }^{7,13,20}$

This work is a theoretical step to understand complex phenomena of incoherency in solids. Finally, we mention two future problems which could be studied numerically in our scheme.

(1) The composition has been taken as a single-order parameter. We should investigate dislocation formation in more general phase separation processes involving an orderdisorder phase transition ${ }^{2,4,16}$ and in diffusionless (Martensitic) structural phase transitions. ${ }^{2,4}$

(2) Dislocations move under applied strain. The motion is complicated when they are coupled with an order parameter and when the time scale of the order parameter is slow. ${ }^{18,29}$

\section{ACKNOWLEDGMENTS}

The authors would like to thank Toshiyuki Koyama for valuable discussions on the incoherency effects in metallic alloys. This work was supported by Grants-in-Aid for Scientific Research and for the 21st Century COE project (Center for Diversity and Universality in Physics) from the Ministry of Education, Culture, Sports, Science and Technology of Japan.

\section{APPENDIX}

Here we assume weak elastic anisotropy and weak elastic inhomogeneity in the coherent condition in two dimensions, supposing shallow quenching. Then we may eliminate the elastic field in terms of $\psi$ using the mechanical equilibrium condition $\nabla \cdot \overleftrightarrow{\sigma}=\mathbf{0}$ in the linear elasticity. We consider the space integral of the last two terms in the free energy density in Eq. (2.3): $\Delta F=\int d \boldsymbol{r}\left[\alpha e_{1} \psi+f_{\mathrm{el}}\right]$. We assume that $\left|\mu_{21}\right|$ and $\left|\mu_{31}\right|$ are much smaller than $L_{0}=K+\mu_{20}$ and that $\xi_{a}$ 
$=2\left(\mu_{20} / \mu_{30}-1\right)$ is small. Then $\Delta F$ may be rewritten as as $^{4,23,24}$

$$
\begin{aligned}
\Delta F= & \int d \boldsymbol{r}\left[-\frac{\alpha^{2}}{2 L_{0}} \psi^{2}+\frac{1}{2} \tau_{\text {cub }}\left|\nabla_{x} \nabla_{y} w\right|^{2}\right]+\int d \boldsymbol{r}\left[g_{2} \psi \mid\left(\nabla_{x}^{2}\right.\right. \\
& \left.\left.-\nabla_{y}^{2}\right)\left.w\right|^{2}+g_{3} \psi\left|\nabla_{x} \nabla_{y} w\right|^{2}\right],
\end{aligned}
$$

where $w$ is obtained from the Laplace equation

$$
\nabla^{2} w=\psi-\langle\psi\rangle,
$$

with $\langle\psi\rangle$ being the average order parameter. In the first line of Eq. (A.1) the bilinear terms are written with

$$
\tau_{\text {cub }}=-\left(2 \alpha^{2} / L_{0}^{2}\right) \mu_{20} \xi_{a} \text {. }
$$

The term proportional to $\tau_{\text {cub }}$ gives rise to anisotropic domains. ${ }^{22}$ The second line consists of the third-order terms with

$$
g_{2}=\mu_{21} \alpha^{2} / 2 L_{0}^{2}, g_{3}=2 \mu_{31} \alpha^{2} / L_{0}^{2} .
$$

The third-order terms are known to give rise to pinning of domain growth (and some frustration effects when $g_{2}$ and $g_{3}$ have different signs). ${ }^{23,24}$

In our simulations we set $\xi_{a}=2(1 / 1.1-1) \cong-0.18$ and $\tau_{\text {cub }} \cong 0.0043 \mu_{20}$, so the domains tend to become square or rectangular with interfaces parallel to the $x$ or $y$ axis. Furthermore, we set $g_{2}=g_{3} / 4 \cong 0.0035 \mu_{20}$. For $\mu_{21} \sim \mu_{31}$ the typical domains in pinned two-phase states $R_{\mathrm{E}}$ is given by ${ }^{23}$

$$
R_{\mathrm{E}} \sim \gamma /\left[\mu_{21}(\Delta c)^{3}\right]
$$

where $\gamma$ is the surface tension and $\Delta c$ is the composition difference between the two phases. Thus $R_{\mathrm{E}}$ decreases as the quenching becomes deeper.
${ }^{1}$ J. W. Cahn, in Critical Phenomena in Alloys, Magnets, and Superconductors, edited by R. I. Jafee et al. (McGraw-Hill, New York, 1971), p. 41.

${ }^{2}$ A. G. Khachaturyan, Theory of Structural Transformations in Solids (Wiley, New York, 1983).

${ }^{3}$ P. Fratzl, O. Penrose, and J. L. Lebowitz, J. Stat. Phys. 95, 1429 (1999).

${ }^{4}$ A. Onuki, Phase Transition Dynamics (Cambridge University Press, Cambridge, 2002).

${ }^{5}$ J. W. Cahn, Acta Metall. 9, 795 (1961).

${ }^{6}$ J. W. Cahn, Acta Metall. 11, 1275 (1963).

${ }^{7}$ J. L. Strudel, in Physical Metallurgy, edited by R. W. Cahn and P. Haasen (North-Holland, Amsterdam, 1996), p. 2106.

${ }^{8}$ E. Nembach, Particle Strengthening of Metals and Alloys (Wiley, New York, 1997).

${ }^{9}$ T. M. Pollock and A. S. Argon, Acta Metall. 40, 1 (1992).

${ }^{10}$ E. A. Marquis and D. N. Seidman, Acta Mater. 48, 3477 (2000); M. J. Jones and F. J. Humphreys, ibid. 51, 2149 (2003); S. Iwamura and Y. Miura, ibid. 52, 591 (2004).

${ }^{11}$ L. D. Landau and E. M. Lifshitz, Theory of Elasticity (Pergamon, New York, 1973).

${ }^{12}$ A. Onuki, Phys. Rev. E 68, 061502 (2003). In this paper a triangular lattice is assumed in two dimensions.

${ }^{13}$ A. H. Cottrell, Dislocations and Plastic Flow in Crystals (Clarendon Press, Oxford, 1953); A. H. Cottrell and M. A. Jaswon, Proc. R. Soc. A 199, 104 (1949).

${ }^{14}$ J. W. Cahn, Acta Metall. 5, 160 (1957).

${ }^{15}$ A. A. Boulbitch and P. Tolédano, Phys. Rev. Lett. 81, 838 (1998).

${ }^{16}$ F. Léonard and R. Desai, Phys. Rev. B 58, 8277 (1988).

${ }^{17}$ S. Y. Hu and L. Q. Chen, Acta Mater. 49, 463 (2001); Comput.
Mater. Sci. 23, 270 (2002).

${ }^{18}$ Y. Wang, D. J. Srolovitz, J. M. Rickman, and R. LeSar, Acta Mater. 48, 2163 (2000).

${ }^{19}$ S. Y. Hu, S. Schmauder, and L. Q. Chen, Phys. Status Solidi B 220, 845 (2000).

${ }^{20}$ A. S. Argon, in Physical Metallurgy, edited by R. W. Cahn and P. Haasen (North-Holland, Amsterdam, 1996), p. 1878, 1958; P. Haasen, ibid. p. 2010.

${ }^{21}$ C. Sagui, A. M. Somoza, and R. C. Desai, Phys. Rev. E 50, 4865 (1994).

${ }^{22}$ H. Nishimori and A. Onuki, Phys. Rev. B 42, 980 (1990).

${ }^{23}$ A. Onuki and H. Nishimori, Phys. Rev. B 43, 13649 (1991); A. Onuki and A. Furukawa, Phys. Rev. Lett. 86, 452 (2001).

${ }^{24}$ D. Orlikowski, C. Sagui, A. M. Somoza, and C. Roland, Phys. Rev. B 59, 8646 (1999); 62, 3160 (2000).

${ }^{25}$ K. Kitahara and M. Imada, Suppl. Prog. Theor. Phys. 64, 65 (1978).

${ }^{26} \mathrm{~K}$. Binder, in Material Sciences and Technology, edited by R. W. Cohen, P. Haasen, and E. J. Kramer (VCH, Weinheim, 1991), Vol. 5.

${ }^{27}$ In simulations with these terms the time mesh size $\Delta t$ must be made very small when dislocations are formed. Such attempts (in the uniaxial deformation case in Figs. 13 and 14) did not essentially alter the results.

${ }^{28}$ The linear elasticity theory (Ref. 11$)$ yields $e_{1}=$ const. $y /\left(x^{2}+y^{2}\right)$ for a single edge dislocation at the origin. The expression for a slip is obtained in the presence of two dislocations with opposite Burgers vectors. See Ref. 10.

${ }^{29}$ A. L. Korzhenevskii, R. Bausch, and R. Schmitz, Phys. Rev. Lett. 91, 236101 (2003). 